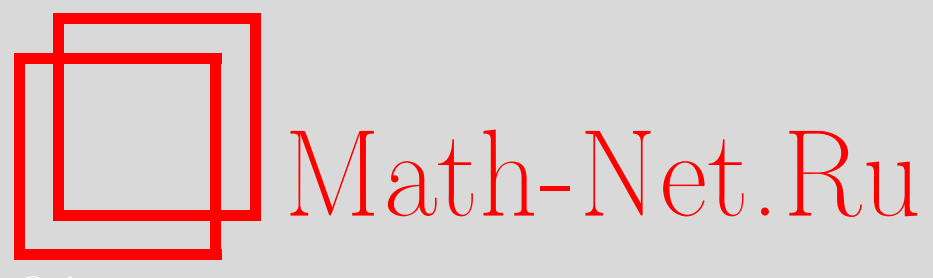

А. Черкун, Течение воды. История, которой 250 лет, Квант, 2020, номер 3, 26-31

DOI: https://doi.org/10.4213/kvant20200303

Использование Общероссийского математического портала Math-Net.Ru подразумевает, что вы прочитали и согласны с пользовательским соглашением http://www.mathnet.ru/rus/agreement

Параметры загрузки:

IP : 54.80 .97 .219

26 апреля 2023 г., $16: 40: 27$

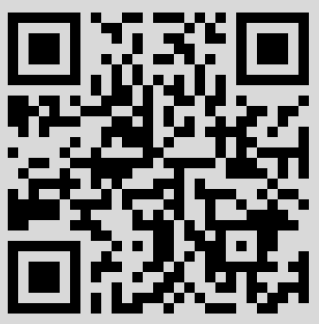




\section{Течение воды. История, \\ которой 250 лет}

A. ЧЕРКУН

Вода - удивительная жидкость. Это важнейшая субстанция живых организмов. Ее прихотливое течение можно долго наблюдать с неослабевающим интересом. Даже простая картина истечения воды через отверстие в сосуде, которую мы не раз видели, таит в себе много неожиданных логических сюрпризов.

В 1974 году в задании Заочной физикотехнической школы (ЗФТШ) мне запомнилась экспериментальная задача. Требовалось в домашних условиях измерить реактивную силу струи воды, вытекающей из бокового отверстия сосуда, и построить график зависимости этой силы от высоты уровня воды над отверстием. Один из вариантов схемы измерения показан на рисунке 1, где сосуд для уменьшения трения располагается на катках или шарах, а реактивная сила

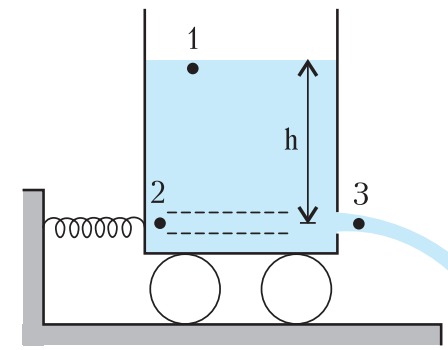

Pnc. 1

измеряется по величине сжатия пружины. Задача интересна тем, что в ней несколько физических понятий соединяются в одно нетривиальное целое, доступное как для теоретического рассмотрения, так и для опытной проверки. Вопросы, которые тогда возникли, прояснились для меня лишь совсем недавно!

DOI: https://doi.org/10.4213/kvant20200303

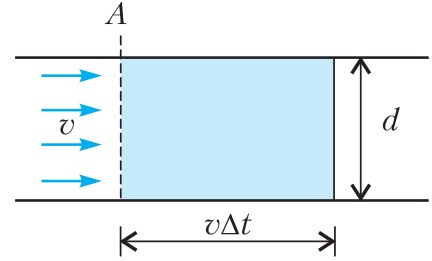

Pис. 2

Вычислим реактивную силу. На рисунке 2 изображен малый горизонтальный участок вытекающей струи. За время $\Delta t$ через сечение $A$ пройдет масса $m=\rho s v \Delta t$, где $v-$ скорость потока, $s=\pi d^{2} / 4$ - площадь сечения, $d$ - диаметр, $\rho$ - плотность воды. Эта масса уносит импульс $p=m v=\rho s v^{2} \Delta t$, который равен импульсу силы, оказываемой сжатой пружиной за то же самое время, т.е. $F \Delta t=p$ (других сил, действующих на систему в горизонтальном направлении, нет). Отсюда находим величину реактивной силы струи:

$$
F_{\mathrm{p}}=F=\rho v^{2} s .
$$

Скорость $v$ можно определить по закону Бернулли (1738 г.), который утверждает, что вдоль линии тока остается постоянной величина

$$
\frac{\rho v^{2}}{2}+\rho g h+p=C .
$$

Здесь $C$ - постоянная, $p$ - давление, $g$ ускорение свободного падения. В точке 1 (см. рис.1), которая находится на высоте $h$, скорость $v=0$, давление $p=p_{\text {a }}$, где $p_{\text {a }}$ атмосферное давление. Значит,

$$
C=\rho g h+p_{\mathrm{a}}=p_{h}+p_{\mathrm{a}},
$$

где $p_{h}=\rho g h-$ гидростатическое давление. В точке $2 v=0, h=0$, следовательно, $p_{2}=C=$ $=p_{h}+p_{\mathrm{a}}$. $\mathrm{B}$ точке $3 p=p_{\mathrm{a}}, h=0$, поэтому $p v_{0}^{2} / 2+p_{\mathrm{a}}=C=p_{h}+p_{\mathrm{a}}$. Тогда для скорости струи $v_{0}$ и для реактивной силы $F_{\mathrm{p}}$ получаем

$$
v_{0}=\sqrt{2 g h} \text { и } F_{\mathrm{p}}=2 p_{h} s .
$$

Да, получен правильный результат, за 250 лет, очевидно, несколько раз проверенный экспериментально. Но можно задать вопрос: а почему реактивная сила в 2 раза больше, чем сила гидростатического давления на площадь $s$ ? Казалось бы, в районе точки 2 на стенку сосуда действует разность сил внутреннего давления $p_{2}$ и наружного атмосфер- 
ного давления $p_{\text {a }}$, равная гидростатическому давлению $p_{h}=\rho g h$, а напротив находится отверстие, где стенки вообще нет. Можно было бы предположить, что на любой высоте силы давления на левую стенку сосуда и на правую стенку сосуда равны друг другу и противоположны по направлению, поэтому они везде компенсируют друг друга, кроме области отверстия справа и области точки 2 слева. В этих двух областях некомпенсированная сила будет равна $F_{S}=p_{h} S$, где $S-$ площадь отверстия. Получается, что в суммарной (векторной) силе давления, действующей на боковые стенки, отсутствует множитель 2 , а в реактивной силе струи он есть, хотя ожидалось равенство $F_{\mathrm{p}}=F_{S}$. Ведь можно сказать, что струя «реактивно» действует на воду в сосуде, вода давит на твердый сосуд, сосуд действует на пружину, и везде должно быть равенство сил, чтобы эти тела не двигались. Например, на сосуд одновременно давит вода и действует пружина. Сила пружины по величине равна реактивной силе струи, значит, суммарная сила давления воды на боковые стенки должна равняться реактивной силе струи, иначе сосуд стал бы двигаться.

Как найти равновесие? Первым шагом в решении этого вопроса стала догадка, что не везде равны давления на левую и правую стенки сосуда (на одной и той же горизонтальной линии вне области отверстия). Дело в том, что вода, подходя к отверстию, как показано стрелками на рисунке 3 , движется рядом с правой стенкой с ненулевой скоростью $v$, которая тем больше, чем ближе отвер-

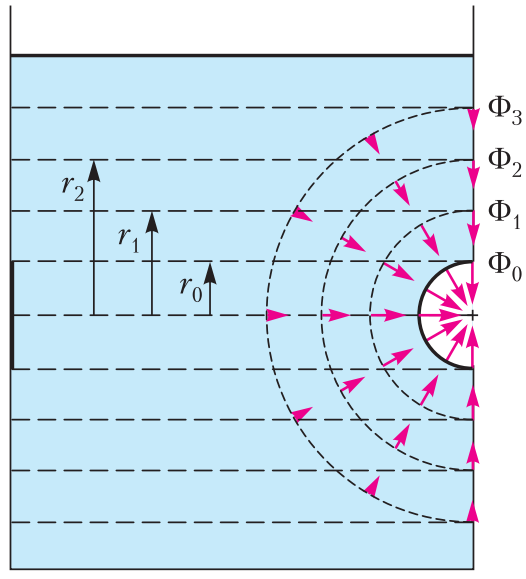

Pис. 3 стие. (На кромке отверстия она становится равной скорости струи $v_{0}$.) Тогда на какойлибо горизонтальной линии вблизи кромки отверстия, например на линии $r_{1}$, на левой стенке $p_{\text {лев }}+\rho g r_{1}=C$ (здесь скорость пренебрежимо мала), а на правой $p_{\text {прав }}+\rho g r_{1}+$ $+\rho v^{2} / 2=C$. Отсюда $p_{\text {лев }}-p_{\text {прав }}=\rho v^{2} / 2$, т.е. здесь давление на левую стенку больше, чем на правую.

Оценим дополнительную некомпенсированную силу $F_{+}$, связанную с этой разностью давлений. Для этого надо знать, как зависит пристеночная скорость $v$ от расстояния $r$ до оси отверстия. Воспользуемся грубой моделью, представленной на рисунке 3. Там символом $\Phi_{0}$ обозначена полусфера радиусом $r_{0}$. Если бы скорость течения везде на этой полусфере равнялась скорости струи $v_{0}$ и была направлена перпендикулярно полусфере, т.е. по линиям ее радиусов, то вся картина течения внутри сосуда стала бы однозначно определенной. Предположим, что мы теми или иными инженерными средствами обеспечили такую ситуацию на полусфеpe $\Phi_{0}$, тогда скорость воды на полусферах $\Phi_{1}, \Phi_{2}, \Phi_{3}$ тоже будет направлена по линиям радиусов и иметь постоянное для данной полусферы значение $v$. Так как через все полусферы проходит один и тот же поток воды, то выполняется равенство $2 \pi r_{0}^{2} \cdot v_{0}=2 \pi r^{2} \cdot v$, где $2 \pi r^{2}-$ площадь поверхности полусферы. Значит, скорость обратно пропорциональна квадрату радиуса:

$$
v=\frac{v_{0} r_{0}^{2}}{r^{2}}
$$

Рассмотрим на правой стенке вокруг отверстия кольцо с меньшим и большим радиусами $r$ и $r+\Delta r$ (рис.4). Если ширина коль-

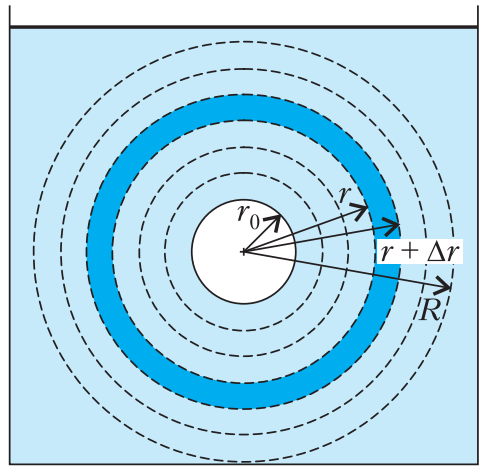

Рис. 4 
ца $\Delta r$ достаточно мала, то пристеночная скорость в пределах этой ширины почти одинаковая и дополнительная сила равна

$$
\begin{aligned}
\Delta F_{+}=\Delta S\left(p_{\text {лев }}-\right. & \left.p_{\text {прав }}\right) \approx \\
& \approx \frac{2 \pi r \Delta r \cdot \rho v^{2}}{2}=\frac{\Delta r \cdot \pi \rho v_{0}^{2} r_{0}^{4}}{r^{3}},
\end{aligned}
$$

где $\Delta S \approx 2 \pi r \cdot \Delta r$. Разобьем правую стенку вокруг отверстия на систему узких колец и просуммируем соответствующие им дополнительные силы $\Delta F_{+}$:

$$
F_{+}=\int_{r_{0}}^{R} d F_{+}=\int_{r_{0}}^{R} \frac{\pi \rho v_{0}^{2} r_{0}^{4}}{r^{3}} d r,
$$

где суммирование ведется от радиуса $r_{0}$ отверстия до достаточно большого радиуса кольца $R$, когда кольцо еще не касается дна сосуда или поверхности воды. Известен и точный результат этого суммирования:

$$
F_{+}=\frac{1}{2} \pi \rho v_{0}^{2} r_{0}^{4}\left(\frac{1}{r_{0}^{2}}-\frac{1}{R^{2}}\right) .
$$

Если радиус отверстия $r_{0}$ достаточно мал по сравнению с глубиной $h$ и расстоянием до дна сосуда, то величиной $1 / R^{2}$ можно пренебречь в сравнении с величиной $1 / r_{0}^{2}$, и тогда получается замечательный результат:

$$
F_{+}=\pi r_{0}^{2} \frac{\rho v_{0}^{2}}{2}=p_{h} S .
$$

Теперь с учетом дополнительной силы $F_{+}$в выражении для суммарной силы давления

$$
F_{S}+F_{+}=2 p_{h} S
$$

появился искомый коэффициент 2.

Казалось бы, равновесие найдено, но в реальности есть важный параметр, который наша модель плохо отражает. При истечении воды через малое (по сравнению с $h$ ) отверстие скорость не может превысить $v_{0}=\sqrt{2 g h}$, тогда поток воды через это отверстие не может превысить $S v_{0}$, а в модели поток равен $2 S v_{0}$, так как площадь полусферы $\Phi_{0}$ в два раза больше площади отверстия $S$. Поэтому в реальности сила $F_{+}$окажется значительно меньше $p_{h} S$.

При дальнейшем поиске равновесия возникает эвристическая догадка, что силу $F_{+}$ можно вообще обратить в ноль! Для этого надо удалить вход отверстия подальше от правой стенки, вставив внутрь перпендикулярно стенке трубочку длиной заметно большей, чем ее диаметр (рис.5). Тогда вдоль

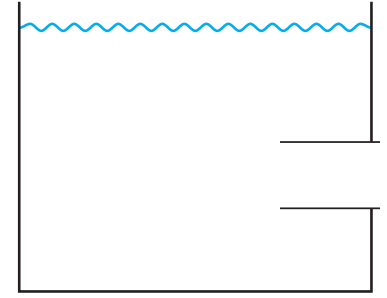

Pис. 5

правой стенки пристеночная скорость $v$ будет пренебрежимо мала, а силы бокового давления воды на трубочку не будут оказывать влияние в горизонтальном направлении измерения реактивной силы.

Поскольку добавочная сила устранилась, приходится вернуться обратно к равенству $F_{\mathrm{p}}=F_{S}$. Это равенство точно расшифровывается так: $2 p_{h} s=p_{h} S$, откуда следует непредвиденный сюрприз: $2 s=S$, т.е. площадь сечения струи в два раза меньше площади отверстия! Оказывается, что диаметры струи и отверстия - это не тождественные, а принципиально разные понятия. Сегодня мне этот факт известен, поэтому для площади струи и площади отверстия сразу были выбраны различные буквы, $s$ и $S$, но это было удивительное открытие, и его эмоционального заряда хватило, чтобы поставить реальный эксперимент. На фотографии, представленной на рисунке 6 , струя воды имеет диаметр примерно в $\sqrt{2}$ раз меньше, чем у трубочки: $20,0 / 14,3 \approx 1,40$. Далее выяснилось, что еще 250 лет назад это открытие сделал французский математик, физик, инженер и морской офицер Жан-Шарль шевалье де Борда, поэтому конструкция трубочки (см. рис.5) названа «насадок Борда».

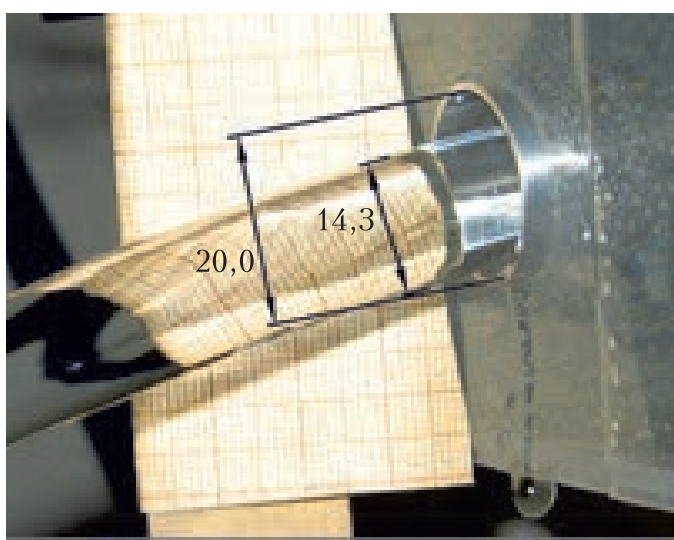

Рис. 6 
Теперь можно вернуться к ситуации обыкновенного отверстия. Если $s$ и $S$ разные, то ответ на начальный вопрос о конструктивном смысле коэффициента 2 найден - действительно реактивная сила равна суммарной силе давления воды на боковые стенки:

$$
F_{\mathrm{p}}=F_{S}+F_{+} \text {, т.е. } 2 p_{h} s=p_{h} S+F_{+} .
$$

И этот ответ состоит из целых трех составляющих: 1) некомпенсированная сила $p_{h} S$ на противоположной от отверстия стенке; 2) дополнительная сила $F_{+}$, связанная с пристеночным течением вокруг отверстия; 3 ) сужение струи, $S>s>S / 2$.

Простой формулы, по которой можно определить либо $s$, либо $F_{+}$, нет. Для их расчета надо написать детальные уравнения гидродинамики и применить технику вычислительной математики. Это другая интересная и сложная задача. Простое применение закона Бернулли в эксперименте дает лишь предсказание скорости струи $v_{0}=\sqrt{2 g h}$, а для прогноза реактивной силы надо еще какимлибо образом узнать диаметр струи, к примеру измерить по фотографии или по расходу воды в секунду. В литературе, например [1, с. 245], указывается, что на опыте для разных высот $h$ при очевидных оговорках наблюдается постоянное соотношение $s / S \approx$ $\approx 0,62$. Следовательно, $F_{+}=2 p_{h} s-p_{h} S \approx$ $\approx 0,24 p_{h} S$, и это в 4 раза меньше, чем по нашей грубой модели. Таким образом, дополнительная сила $F_{+}$становится экспериментально определенной величиной.

Какие физические моменты в процессе сужения потока можно себе представить? На рисунке 7 показаны линии тока воды. На самой крайней линии, обозначенной цифрой 1, применение закона Бернулли дает

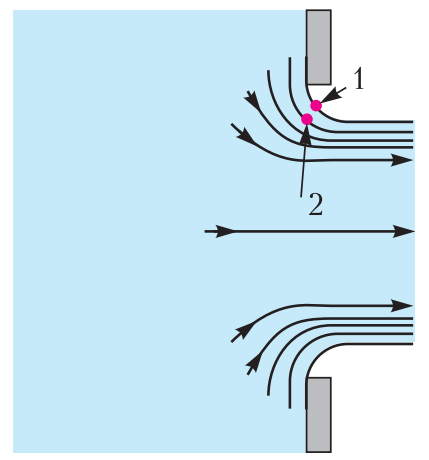

PUC. 7 «всюду» постоянную скорость $v_{0}=\sqrt{2 g h}$. Эта линия является продолжением пристеночного течения, которое отвечало за появление дополнительной силы $F_{+}$, поэтому крайняя линия разворачивается на $90^{\circ}$, меняя вертикальное направление на горизонтальное. Криволинейное движение с постоянной скоростью происходит с центростремительным ускорением, которое должно вызываться соответствующей силой, поэтому на соседней линии тока под номером 2 в точке 2 должно быть большее давление, чем в точке 1, следовательно, в точке 2 скорость меньше (!), чем $v_{0}$. По мере удаления от отверстия скорость и давление на линии 2 постепенно приближаются к постоянной скорости и атмосферному давлению $p_{\text {a }}$ на линии 1 .

Оценим теперь влияние вязкости жидкости. Рассмотрим пластину, которая поддерживается на постоянном расстоянии $L$ от неподвижной стенки и тянется с постоянной скоростью v (рис.8). В зазоре между пласти-

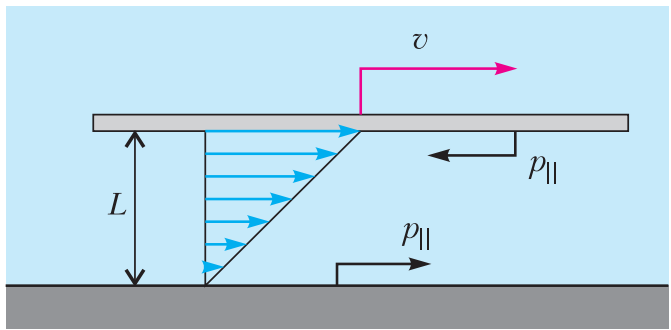

Pис. 8

ной и стенкой возникает послойное движение жидкости такое, что жидкость «прилипает» к стенке и имеет нулевую скорость рядом со стенкой, но чем дальше от стенки, тем скорость становится больше и больше, пока не сравняется со скоростью пластины, т.е. пока жидкость не «прилипнет» к пластине. При этом на поверхности стенки и пластины будут действовать удельные вязкие силы $p_{\|}$, направленные параллельно поверхностям. Удельная сила имеет размерность давления и равна $p_{\|}=\eta v / L$, где $\eta-$ коэффициент динамической вязкости жидкости. Для воды при комнатной температуре $\eta \approx 0,001$ Па $\cdot$ с. Для грубой оценки влияния вязкости обычно вместо $L$ берется характерный размер потока, например радиус отверстия $r_{0}$, и полученное таким образом вязкое давление $p_{\|}$сравнивают с каким-либо характерным давлением, например со скоростным 
напором $p_{v}=\rho v^{2} / 2$. Безразмерное отношение $2 p_{v} / p_{\|}=\rho v L / \eta$ называется числом Рейнольдса и обозначается Re. Для нашего реального эксперимента, в котором $r_{0}=1 \mathrm{~cm}$, $h=20 \mathrm{cм}, g=9,81 \mathrm{~m} / \mathrm{c}^{2}, \rho=1000 \mathrm{\kappa г} / \mathrm{м}^{3}, v_{0}=$ $=\sqrt{2 g h} \approx 2 \mathrm{м} / \mathrm{c}$, число Рейнольдса $\mathrm{Re} \approx$ $\approx 20000$. Значит, характерное вязкое трение здесь меньше характерного динамического давления во многие тысячи раз, поэтому можно полагать, что описание линий тока (см. рис.7) близко к реальности. Правда в реальности возникает сложная структурная картина, связанная с вязким «прилипанием» потока к стенке, но характерная толщина и другие размеры этой структуры относительно малы.

Неожиданно обнаружилось, что в нескольких современных учебниках приводится отличная от рисунка 7 картина. Так, на рисунке 9, взятом из книги [2, с. 88] (подобный

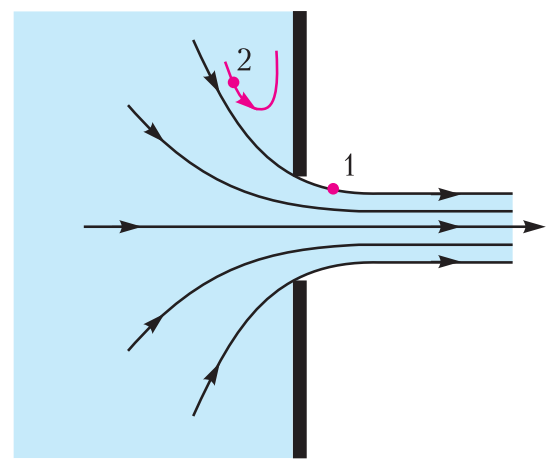

Pис. 9

рисунок есть также в книге [3, с. 496]), крайняя линия тока, обозначенная 1, не касается вертикальной стенки. Тогда возникает вопрос: что происходит в точке 2 чуть выше линии тока 1? Если в точке 2 скорость ненулевая, то соответствующая линия тока не выйдет через отверстие, так как крайняя линия 1 уже есть. Значит, эта линия должна развернуться и замкнуться в кольцевой поток. Если же в точке 2 скорость равна нулю, то это означает резкий скачок скорости непосредственно рядом и вдоль всей линии 1. Оба случая представляются маловероятными (хотя гидродинамика богата сюрпризами).

Еще более рельефно выступает вопрос о картине течения при заходе в насадок Борда. На рисунке 10 выше и ниже средней линии даны два возможных варианта течения. Пер-

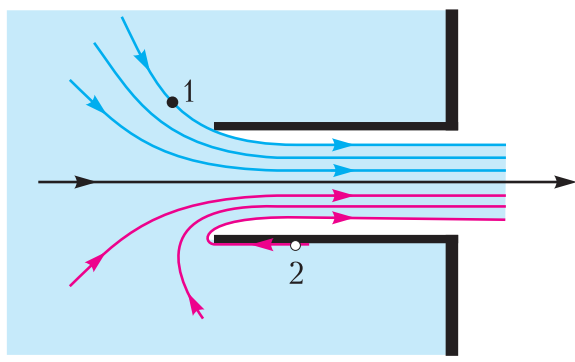

Pис. 10

вый соответствует рисунку из книги [2, c. 88] (и похожий есть в книге [1, с. 245]), а второй составлен так, чтобы учесть пристеночное течение, обозначенное крайней линией 2. Здесь эта линия совершает разворот на $180^{\circ}$, т.е. меняет направление на противоположное. (Тогда как крайняя линия 1 поворачивает лишь на $63^{\circ}$.)

Для выяснения реальной картины был проведен соответствующий опыт. В стенку прямоугольной 15-литровой емкости было встроено качественное стеклянное окно диаметром 8 см прямо напротив входа воды в насадок Борда. Через это окно цифровым фотоаппаратом с лампой-вспышкой получено фото, приведенное на рисунке 11. На

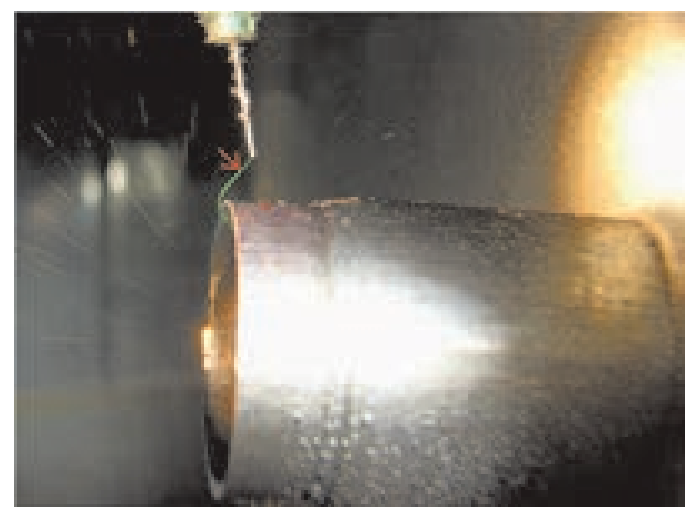

PUC. 11

фото в насадок Борда 1 втекает поток. Внутренний диаметр трубочки 20 мм, а внешний диаметр рядом со входом 20,3 мм. Из медицинской иголки 2 вытекают чернила, и их тонкая зеленая траектория, обозначенная стрелкой, показывает линию тока, которая разворачивается приблизительно на $135^{\circ}$, что больше соответствует варианту ниже средней линии на рисунке 10.

Неожиданно лампа-вспышка и случайные пузырьки воздуха помогли увидеть допол- 
нительные детали. Маленькие пузырьки захватываются мощным течением и движутся почти по линиям тока. За время, что длится вспышка, каждый пузырек, попавший в фокус фотоаппарата, оставляет на фотографии протяженный след, указывая линию тока. Этот след тем длиннее, чем больше скорость потока, т.е. дополнительно получается информация о скорости. Цифрой 3 на рисунке 11 отмечена группа коротких черточек-следов, которые также указывают на наличие течения с внешней стороны насадка Борда навстречу центральному потоку. Образно говоря, этот внешний поток «сталкивается» с центральным потоком.

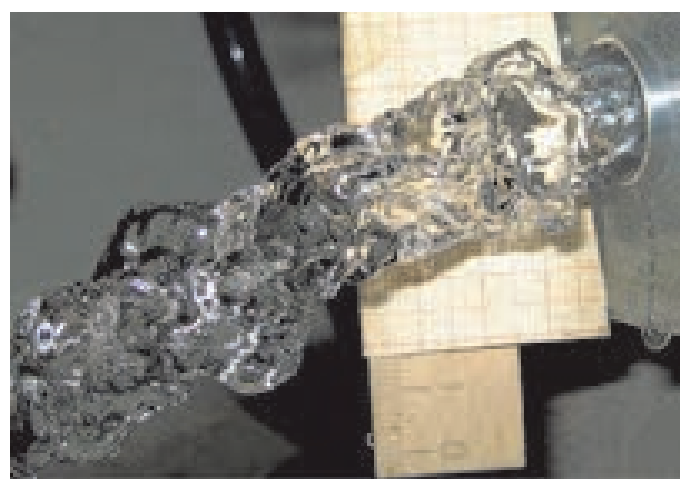

Pис. 12

Еще один яркий сюрприз зафиксирован на фотографии, представленной на рисунке 12. Оказывается, при одной и той же глубине $h$ в одной и той же трубке Борда бывают два совершенно разных устойчивых режима течения: ламинарный, как на рисунке 6, и турбулентный, как на рисунке 12! Какой режим возникнет, зависит от стартовых условий. Если резиновую пробку (с небольшой боковой конусностью) на старте вынимать из трубки с наружной стороны, то возникает турбулентный режим, а если с внутренней стороны в толще воды, то возникает ламинарный режим. Чтобы в последнем случае не возмущать воду рукой, резиновая пробка крепится к длинному (вертикальному) стержню посредством прямой гибко- упругой проволоки, пронизывающей пробку вдоль центральной оси. Если просто смотреть на турбулентный поток, то он белый, шумный, беспокойный, брызгающий, но лампа-вспышка «остановила» этот внешний хаос, и открылась хрустальная прозрачность, плавность и какая-то внутренняя неуловимая сущность. Теперь поток касается стенок трубки, и расход воды увеличивается (!) по сравнению с ламинарным режимом.

Математическое моделирование турбулентности до сих пор остается интеллектуальным вызовом. Простые домашние эксперименты, наподобие тех, что представлены в статье, могут дать не только ответ на сотни собственных вопросов, но и послужить основой какой-либо новой концепции турбулентности. И вообще, гидро- или аэродинамику стоит воспринимать как интереснейшую область для творческого осмысления.

Снова вернемся к опытам с насадком Борда. Если взять не круглую трубку, а трубку (из жести) с прямоугольным 1:3 сечением, то соотношение $2 s=S$ сохраняется. А как будет выглядеть струя? Удастся ли вообще получить ламинарный режим? Или если случайные пузырьки воздуха оказались столь эффективны для визуализации картины течения, то, может быть, стоит пузырьки создавать искусственно? Например, можно по одной медицинской иголке подавать раствор соды $\mathrm{NaHCO}_{3}$, а по соседней иголке - paствор лимонной кислоты, тогда в смеси двух потоков должны образовываться пузырьки углекислого газа $\mathrm{CO}_{2}$.

Сотни вопросов и сотни вариантов ответов... Твори, выдумывай, пробуй!

\section{Литература}

1. Р.Фейнман, Р.Лейтон, М. Сэндс. Фейнмановские лекции по физике. Выпуск 7. М.: Мир, 1966.

2. T.Е.Фабер. Гидроаэродинамика. - М.: Постмаркет, 2001.

3. Д.В.Сивухин. Общий курс физики. Том 1. - М.: Физматлит, 2016. 